\title{
Association of Dental Pulp Stem Cells and Ricinus Bone Compound in a Model of Bone Defect
}

\author{
Alan Araújo de Jesus ${ }^{1,2}$, Adriano Araújo de Jesus ${ }^{1,2}$, Flávia Oliveira de Limaa ${ }^{1,3}$, Luiz Antônio Rodrigues de Freitas ${ }^{1}$, \\ Cássio Santana Meira ${ }^{1,4}$, Ricardo Ribeiro dos Santos ${ }^{1,2,4}$, Milena Botelho Pereira Soares ${ }^{1,2,4^{*}}$ \\ ${ }^{1}$ Gonçalo Moniz Institute, Oswaldo Cruz Foundation (FIOCRUZ), Salvador, Bahia; ${ }^{2}$ Centre of Biotechnology and \\ Cellular Therapy, São Rafael Hospital; ${ }^{3}$ State University of Feira de Santana, Feira de Santana, Bahia; ${ }^{4}$ ISI (SENAI \\ Institute for Innovation in Advanced Health Systems) /SENAI CIMATEC; Salvador, Bahia, Brazil
}

\begin{abstract}
Autogenous bone grafting is needed in some bone tissue defects; however, it causes secondary surgical wounds and morbidity. Tissue bioengineering may be an alternative approach for bone regeneration. Here we investigated the osteogenic potential of dental pulp stem cells from deciduous teeth (DPSC) in association with a Ricinus bone compound (RBC) in a model of bone defect. The influence of the biomaterial RBC on the proliferation and osteogenic differentiation of DPSC was assessed in vitro by MTT metabolism and alizarin red staining, respectively. The morphologic analysis was performed using the optic and scanning electron (SEM) microscopies. For the in vivo study, 54 Wistar rats submitted to calvarial defects were filled with RBC or RBC+DPSC. A control group had the defects filled only with blood clots. Analyses were performed 15, 30 and 60 days after treatment using digital radiography, optical microscopy, SEM and chemical analysis by electron dispersive spectroscopy. The Ricinus bone compound (RBC) did not inhibit the osteogenic differentiation in vitro. No spontaneous regeneration was observed in the control group. The area of the calvarial defect of the RBC+DPSC group showed greater radiopacity on day 15 . The $\mathrm{RBC}$ presented no reabsorption, was biocompatible and showed osteointegration, working as a mechanical filling. Only sparse ossification areas were found and those were larger and more developed on the RBC+DPSC group when compared to animals treated only with RBC. RBC in association with DPSC is a promising combination for applications in bone regeneration.

Keywords: Bone Defect. Dental Pulp. Stem Cells. Tissue Therapy.

Abbreviations: CV: coefficient of variation; DMEM: Dulbecco's modified Eagle medium DPSC: dental pulp stem cells; EDS: electron dispersive spectroscopy; FBS: fetal bovine serum; MTT: 3-[4, 5-dimethylthiazolyl-il]2,5-diphenyltetrazolium bromide; RBC: Ricinus bone compound; SEM: scanning electron microscopy.
\end{abstract}

\section{Introduction}

The bone is a very dynamic tissue with good regenerative capacity, but when significant tissue loss occurs due to traumas or injuries, graft replacement is needed. Autogenous grafts, also known as autografts, are the golden standard choice of treatment; however, autograft replacement requires a donor area, leading to an extra surgical wound, increasing consequently time and complexity of the surgical procedure and

\footnotetext{
Received on 20 July 2020; revised 19 August 2020.

Address for correspondence: Milena Botelho Pereira Soares. Instituto Gonçalo Moniz, Fundação Oswaldo Cruz, Rua Waldemar Falcão, 121, Candeal, Salvador, BA, Brazil. CEP: 40296-710. Phone: (55) (71) 3176-2260; Fax: (55) (71) 3176-2272. E-mail address: milena@bahia.fiocruz.br. DOI 10.34178/jbth.v3i3.129.
}

J Bioeng. Tech. Appl. Health 2020;3(3):267-278.

C 2020 by SENAI CIMATEC. All rights reserved. the risk of infections, worsening postoperative recovery and raising costs. Moreover, there are limitations to obtain accessible donor areas with enough good-quality bone tissue [1]. Biomaterials are an alternative for autogenous bone graft and its inherent problems, and different biomaterials have been used for bone lesion regeneration. The available materials, however, do not have all properties to allow their use in all types of defects [2]. A promising perspective for avoiding the disadvantages of autogenous grafts would be supplying already available biomaterials with cellular components. The absence of cellular components is the main deficiency of this type of material. Combining them to previously collected and cultured stem cells from the patient may generate a cellularized microenvironment similar to a natural bone, allowing total integration, graft vascularization, and, therefore, bone regeneration [3]. 
Deciduous teeth pulp is an accessible source of stem cells obtainable from non-vital organs, normally disposed of after the exfoliation phase for the eruption of permanent teeth [4]. Dental pulp stem cells (DPSC) have proliferation capacity and a promising tissue regeneration induction potential previously shown $[4,5]$.

The objective of this study was to assess the in vitro and in vivo osteogenic potential of DPSC when associated with the biomaterial Ricinus bone compound in vitro and in vivo in a model of bone regeneration in rats.

\section{Materials and Methods}

\section{$\underline{\text { Biomaterials }}$}

We used in the study the biomaterial Ricinus bone compound (RBC; Poliquil Araraquara Polímeros Químicos, Araraquara, Brazil), a polyurethane composed of a pre-polymer derived from isocyanate, a polyester polyol derived from castor oil and calcium carbonate $(0.65 / 1)$ and calcium carbonate $50 \%$ (which allows the formation of pores and gives a better standard of resistance and elasticity in comparison to the bone tissue), with granule particles of about $500 \mu \mathrm{m}$. To assess the biomaterial by scanning electron microscopy (SEM), a JSM 6390LV microscope (JEOL Ltd., Tokyo, Japan) was used. Particles of the biomaterial were bound to stubs with carbon fiber and subjected to a sputter coating device SCD 050 (BAL-TEC AG, Liechtenstein, Switzerland) for deposition of an ultrathin layer of gold, turning the samples electrically conductive.

\section{Dental Pulp Stem Cell (DPSC) Culture}

We used a previously characterized human cell line obtained from pulp tissue removed from a deciduous tooth extracted near the natural exfoliation phase [6]. DPSC were cultured in tissue culture flasks containing Dulbecco's modified Eagle medium (DMEM; Life Technologies, GIBCO-BRL, Gaithersburg, MD) supplemented with $10 \%$ fetal bovine serum (FBS; Cultilab, Campinas, Brazil) at $37^{\circ} \mathrm{C}$ and $5 \% \mathrm{CO}_{2}$. The medium was renewed every three days during approximately 10 days when the culture reached $80-90 \%$ confluence. Cells were detached using $0.25 \%$ trypsin (Invitrogen Corporation, São Paulo, Brazil). After washing with serum-free medium, cell suspensions were counted using a hemocytometer and used for in vitro and in vivo studies.

\section{In vitro Study}

\section{Assessment of Cell Proliferation}

An extract of the biomaterial was obtained after incubation in DMEM (200 mg of biomaterial for $1 \mathrm{ml}$ of medium) for 24 hours at $37^{\circ} \mathrm{C}$. After incubation, extracts were subjected to centrifugation for collection of the supernatant. DPSC $\left(10^{4} /\right.$ well) were plated in 96-well plates. Cells were incubated for 24 hours $\left(5 \% \mathrm{CO}_{2}, 37^{\circ} \mathrm{C}\right.$, $>90 \%$ humidity), allowing them to adhere and form a semi-confluent layer. Next, cells were incubated in the absence or in the presence of the extract of the biomaterial (diluted in complete DMEM medium at 1:5, 1:100 and 1:250), in triplicate. Qualitative analysis was performed by optical microscopy. Quantitative analysis was done by the MTT (3-[4, 5-dimethylthiazolyl-il]2,5-diphenyltetrazolium bromide; Sigma-Aldrich, St. Louis, MO) assay, 48 hours later. Plates were incubated for $4 \mathrm{~h}$ at $37^{\circ} \mathrm{C}$ when the reagent was removed and $0.1 \mathrm{~N}$ of hydrochloric acid diluted in isopropanol was added. Absorbance was determined 30 minutes and 4 days later using a spectrophotometer Spectramax 190 (Molecular Devices, Sunnyvale, CA) at $570 \mathrm{~nm}$.

\section{Osteogenic Differentiation}

For osteogenic induction, DPSCs were cultured in 24-well plates with DMEM medium supplemented with $10 \%$ FBS for two days, until $50 \%$ confluence was reached. Medium for osteogenic differentiation (DMEM, 10\% FBS, $100 \mathrm{nM}$ dexamethasone, $0.05 \mathrm{M}$ L-ascorbic acid 
2-phosphate, and $10 \mathrm{mM} \beta$-glycerophosphate; all from Sigma-Aldrich) was added, with or without the conditioned media of the biomaterial. Cultures were then incubated at $37^{\circ} \mathrm{C}$ and $5 \% \mathrm{CO}_{2}$ for 21 days. A control culture of DPSC was maintained only with DMEM with $10 \%$ FBS. Experiments were carried out in triplicate and the media was replaced every 3 days. Cultures were then washed with PBS, fixed using 4\% paraformaldehyde (Electron Microscopy Sciences, Hatfield, PA) for 30 minutes, washed twice with distilled water, and then stained with alizarin red (Sigma-Aldrich) at $2 \%$ for 3 minutes. After removal of the dye, cultures were washed three times with distilled water. Digital images of the calcium deposits were used for quantitative analysis, to estimate the percentage of the stained area in each well using the Image-pro program (Media Cybernetics, Rockville, MD).

\section{Assessment in vivo}

\section{Animals}

Animals were provided and maintained at the Animal Facility of Instituto Gonçalo Moniz/ FIOCRUZ. Fifty-four adult male animals (Rattus norvegicus, strain Wistar) were used, weighing between 400 and $450 \mathrm{~g}$, between 5 and 6 months of age. Animals were randomly selected and equally divided into three groups with three time-points each. All experiments followed the regulation for use of animals in experiments and were approved by a local Ethical Committee for Animal Use.

\section{Surgical Procedure}

Animals underwent general anesthesia by intraperitoneal injection with xylazine hydrochloride (5 to $10 \mathrm{mg} / \mathrm{kg}$ ) and ketamine hydrochloride (50 to $75 \mathrm{mg} / \mathrm{kg}$ ). After trichotomy, the periosteum was detached through a linear incision to give access to the bone tissue. The calvarial bone defect was created in the median portion using a $7 \mathrm{~mm}$ trephine drill (FTR06, SIN, São Paulo, Brazil), coupled to a counter-angle with $1: 16$ reduction, and activated by a low rotational speed surgical motor (Implantmed Si - $923 \mathrm{WH}$, Bürmoos, Austria), under constant irrigation with sterile saline solution. Grafts were executed at this moment. Three types of treatments were carried out: a control group where defects were filled only with blood clots (control), with $25 \mathrm{mg}$ of RBC homogenized with a blood clot (RBC group) or with $25 \mathrm{mg}$ of $\mathrm{RBC}$ homogenized with blood clot plus $2 \times 10^{6}$ cells (RBC+DPSC group). Animals were euthanized on days 15, 30 and 60 after surgery. Standard size bone fragments of the defects were removed, including a safety margin around the defect. For the removal of bone fragments, a low rotational diamond drill was used under constant irrigation with a saline solution. For histological procedures, the obtained bone pieces were kept in $4 \%$ buffered paraformaldehyde for seven days under refrigeration. After fixation, all bone pieces were photographed $(100 \mathrm{~mm}$ macro lens, Canon Inc., Tokyo, Japan) on its inferior and superior view for registry of integrity and further macroscopic analysis.

\section{Digital Radiographic Analysis}

Samples were placed in a transversal position on the image slides for periapical radiographs on the digital radiography system CDR USB (Sirona Dental, Inc., Long Island City, N.Y.). An aluminum penetrometer of 5 degrees with a $1 \mathrm{~mm}$ increment was added to the system. The radiographic device $(70 \mathrm{kV})$ was programmed for an exposition time of 0.02 seconds, with a focal distance of $10 \mathrm{~cm}$. The software Photoshop ${ }^{\circledR}$ (Adobe Systems Incorporated, San Jose, CA) was used for the correction of brightness in the images, using the penetrometer as a parameter. To quantify the tissue radiopacity, the mean and standard deviation of the gray intensities was measured using the Image Tool ${ }^{\circledR}$ software (University of Texas Health Science Center, San Antonio, TX). The coefficient of variation (CV) of the same area was also calculated. For each radiograph, two circumferences of the same diameter were established: one on the defect place and the other 
outside any area with residual bone tissue. This area was set as an individual negative control for each image. The final result was obtained by subtracting the value from each control area from its defect area. Defect areas were not compared among each other, since there is a difference in contrast between the images, which could create a bias in the absolute value for direct comparison between samples, even taking into account the brightness correction previously performed. To eliminate the possible influence of the biomaterial on the results, radiographs of the biomaterial alone were carried out separately, following the same protocol.

\section{Histological Analysis by Optic Microscopy}

After fixation, samples were decalcified in $7 \%$ nitric acid for 72 hours. Samples were then transversally cut through their large diameter, subjected to the standard protocol for paraffinembedded tissue, and sliced with a microtome RM 2125 (Leica Biosystems, Wetzlar, Germany) in 5 $\mu \mathrm{m}$ thick transversal sections. Slices were stained with conventional hematoxylin and eosin staining. Tissue regeneration was observed under an optical microscope with different resolutions and assessments done by descriptive analysis. Several aspects were reported, such as the presence of inflammatory cells, the number of collagenous fibers, contact interface between the biomaterial and bone tissue, degradation of the particles of the biomaterials and osteogenesis.

\section{$\underline{\text { Scanning Electron Microscopy Analysis (SEM) }}$}

The analysis by SEM was carried out in the group of animals sacrificed at 60 days since it was the longest period for bone regeneration studied. For fixation, samples were immersed in $4 \%$ paraformaldehyde, $2.5 \%$ glutaraldehyde diluted in $0.1 \mathrm{M}$ sodium cacodylate buffer. After fixation, pieces were cut in the middle through the larger diameter of the defect. Half of each sample was decalcified and prepared for optic microscopy and the other half was prepared for SEM. The samples prepared for SEM were subjected to dehydration through a sequence of alcohol baths in different concentrations using a critical point drier (CPD 030, BAL-TEC), and to metallization by a sputter coating, as described above. For comparison, assessment of integer bone fragments from healthy animals was also carried out using the same size as for the defects in the test samples.

\section{Chemical Analysis by Electron Dispersive} Spectroscopy (EDS)

Chemical compounds present in the samples were observed using the EDS Nanotrace system TNJEM1230-3NUS (Thermo Electron Corporation, Waltham, MA). The distance from samples to the detector was $10 \mathrm{~mm}$ and $15 \mathrm{kV}$. Sample preparation followed the same protocol as for SEM.

\section{$\underline{\text { Statistical Analysis }}$}

Statistical analysis was carried out using GraphPad Prism ${ }^{\circledR}$ software version 5.01 (GraphPad Software, Inc., San Diego, CA). Mean differences were subjected to variance analysis using one-way ANOVA with the Bonferroni test for multiple comparisons. P-value was considered significant when lower than 0.05 .

\section{Results}

The biomaterial $\mathrm{RBC}$ presented particles up to $800 \mu \mathrm{m}$ in size, with an irregular shape, broad concavities and spiked edges with a slightly rough surface, except for the areas where fractures occurred during the grinding process (Figures $1 \mathrm{~A}$ and B). DPSC were cultured in complete DMEM medium in the absence or presence of RBC extract. After two days of culture in RBC extract at 1:5 dilution, we observed a reduction in cell viability. RBC at 1:250 did not inhibit cell proliferation, as shown by MTT assay (Figure 1C). Based on these results, we selected 1:250 dilution of the extract to be used. 
DPSC cultured in osteogenic medium in the absence or presence of RBC extract were analyzed to detect calcium depositions by alizarin red staining 21 days after culture. A statistically significant difference in the percentage of alizarin red-stained area was observed in the groups cultured in the osteogenic medium when compared to the control cultures in DMEM medium alone (Figure1D). No differences between cultures incubated without or with the $\mathrm{RBC}$ extract in osteogenic-inducing conditions were found.

The model for cranial vault defect provided areas with a $7 \mathrm{~mm}$ diameter with no spontaneous regeneration until the end of the experimental procedures. Macroscopic assessment of the pieces 15 days after defect induction showed a better regeneration of the periosteum and endosteum than in the RBC+DPSC group than in the RBC group, as shown by an increased tissue formation in the regeneration area. The control group had reduced regeneration (Figure 2).

Quantification by analysis of digital X-ray images at day 15 revealed a statistically significant difference in the mean of gray intensity levels (Figure 3A) and coefficient of variation (Figure 3B) among the three groups. The control group showed no radiopacity of the tissue. RBC+DPSC group obtained higher values for gray level and $\mathrm{CV}$ than the RBC group. Analysis carried out on days 30 and 60 revealed that all groups increased in value over time, with no significant difference between the gray levels in groups RBC and $\mathrm{RBC}+\mathrm{DPSC}(\mathrm{p}>0.05)$, while both groups had significantly higher levels $(\mathrm{p}<0.05)$ than the control group (data not shown).

Histological analyses showed a marked decrease in thickness on the defect area in the control group (Figure 4A). This area was filled

Figure 1. Ultra-structural analysis of the biomaterial by scanning electron microscopy.

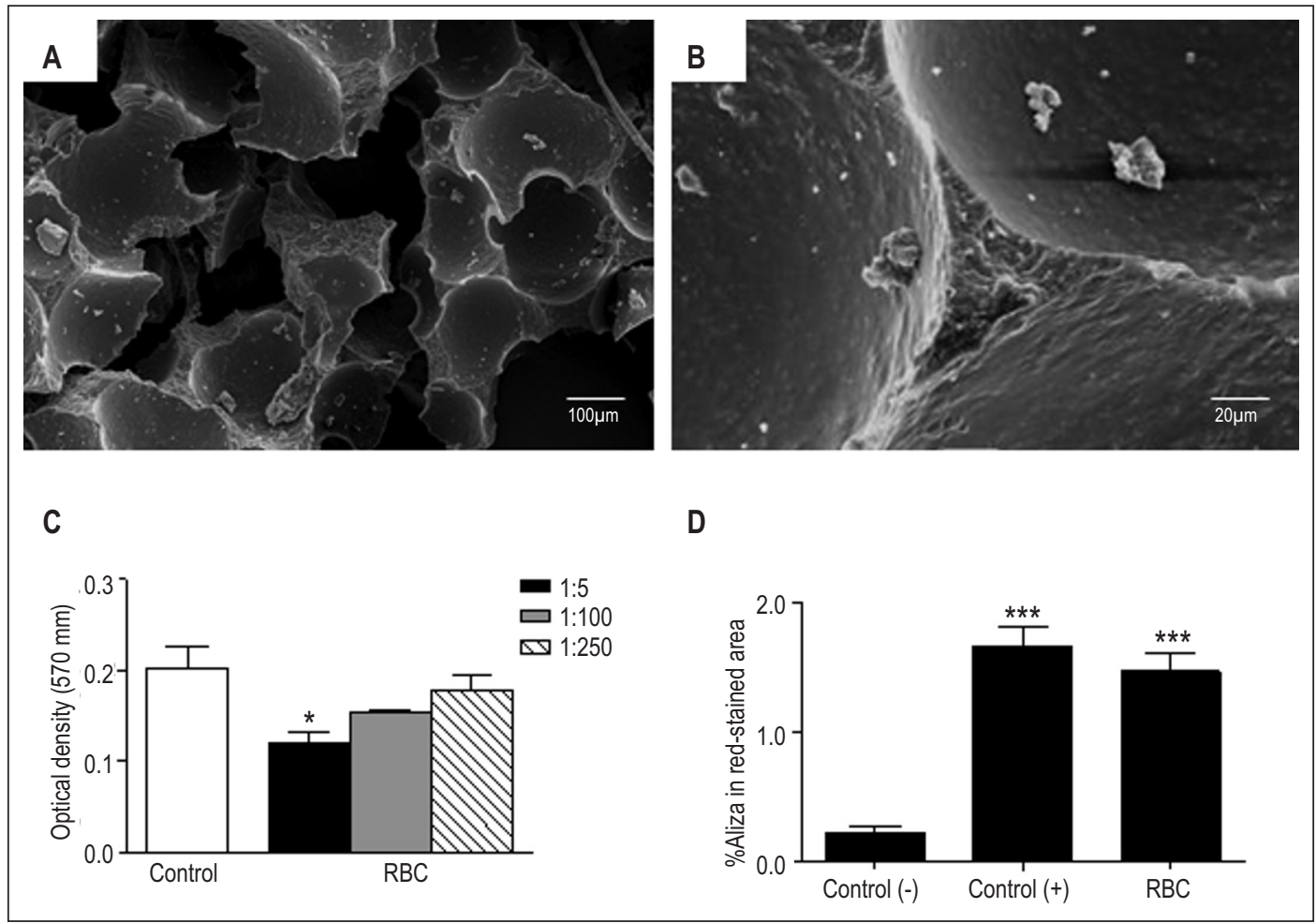

(A) RBC particles of different sizes, with sharp ridges. (B) The detail in higher magnification showing differences in the surface roughness between areas of convexities and areas of fragmentation. (C) RBC at 1:250 did not inhibit cell proliferation, (D) No differences in osteogenic differentiation between cultures incubated without or with the RBC extract. 
Figure 2. Macroscopic assessment of the pieces 15 days after defect induction.
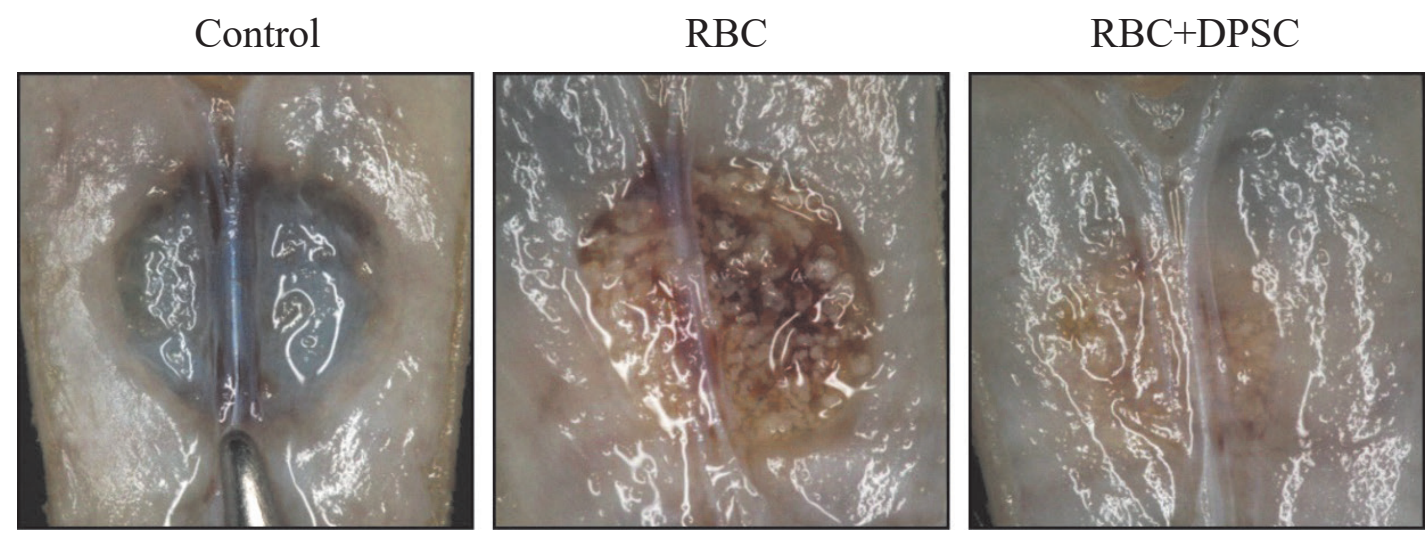

(A) Animals where defects were filled only with blood clots (control). (B) Animals where defects were filied with $25 \mathrm{mg}$ of RBC homogenized with a blood clot (RBC group). (C) Animals where defects were filled with $25 \mathrm{mg}$ of RBC homogenized with blood clot plus $2 \times 10^{6}$ cells (RBC+DPSC group).

Figure 3. Digital radiographic analysis in bone defects. Three types of treatments were carried out: a control group where defects were filled with a blood clot (control), with $25 \mathrm{mg}$ of RBC homogenized with a blood clot (RBC group), or with $25 \mathrm{mg}$ of RBC homogenized with blood clot plus $2 \times 10^{6}$ DPSC (RBC+DPSC group). Groups of rats were euthanized on day 15 for analyses.

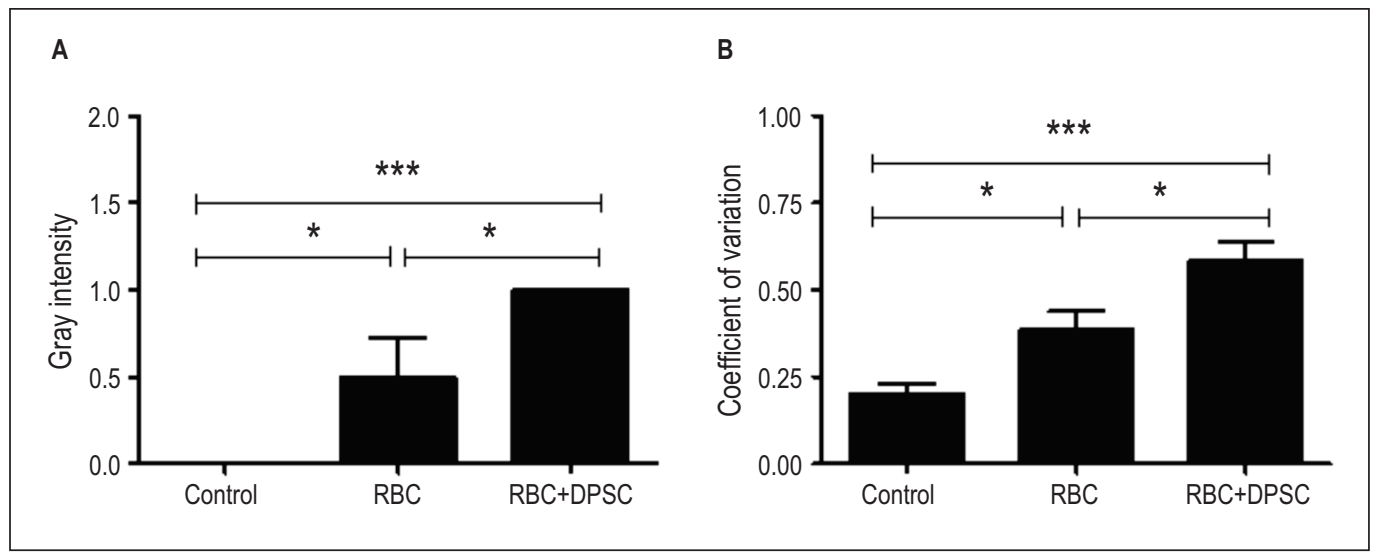

(A) Gray levels of the defect region in the three studied groups. (B) Coefficient of variation of the defect region in the three studied groups. The values represent the mean \pm SEM from the percentages of 6 animals/group. ${ }^{*} \mathrm{p}<0.05$ and $* * * p<0.001$.

in with fibrous connective tissues presenting fusiform cells arranged horizontally, progressing from loose on day 15 to dense on day 60 . Several blood vessels were found near the margin of the defects in all-time points with a greater number at day 60 when vessels were found spread into the center of the defects. Regarding bone regeneration, the defects were critical as they would not regenerate spontaneously. No significant signs of an inflammatory process, either chronic or acute, resulted from the surgical trauma, were found at the time points analyzed (Figure 4A). On the RBC samples, no signs of particle degradation were found at the studied time points, and the presence of the biomaterial kept the thickness of the defect (Figure 4B). The ridges of the particles 
seemed to hamper contact between the connective tissue and the material's surface (Figure 4C). Some giant multinucleated cells were sparsely found making contact with some particles; however, no inflammatory infiltrate was found, suggesting biocompatibility of the material. Regarding bone regeneration, ossification of some areas was occasionally found at days 30 and 60 (Figure $4 D)$. In those regenerating areas, it was possible to identify a layer of osteoblasts surrounding the region and osteocytes in their center, suggesting regeneration progression was taking place. We observed osseointegration between the particles of the biomaterials and newly formed bone tissue in the groups at day 60 (Figure 4E) and the presence of mineral tissue in close contact with the particles of the biomaterial. Blood vessels were found spread widely over the defect in all-time points, and in a greater frequency at day 60 . The characteristics of the RBC+DPSC group were similar to the RBC group, yet larger ossified regions with maturation aspects were found already on day 15 (Figures 4F and $\mathrm{G}$ ). In both groups where the biomaterial was used, it was possible to correlate ossified regions with the proximity of a larger number and blood vessels, especially those of larger calipers. Bone regeneration was restricted to incidental (random) areas and most particles were still surrounded by fibrous connective tissue (Figure 4H).

The analysis by SEM enabled assessment of the microarchitecture of the defects, and validation of the findings observed by optic microscopies, such as a decrease in thickness on the defect area in the control group (Figure 5A) and the recovery of the thickness by the biomaterials (Figure $5 \mathrm{~B}$ ), properties of the edges (Figure 5C), bone regeneration (Figure 5D) and distribution of blood vessels (Figure 5E). Samples exhibited tissue with fibrous aspect portraying the lack of tissue regeneration. However, regions where fibers were arranged in bundles, which might have progressed to bone tissue over time, were also found (Figure $5 \mathrm{~F})$.

At day 60 the biomaterial did not sustain changes in its form after making contact with the tissue, and the particles kept their aspect, with spiked edges, hampering a full contact between the material's surface and the newly-formed tissue (Figure 5G). The assessment of intact bone fragments showed that their thickness (Figure 5H) were similar to those of the groups with biomaterials, confirming their role as filling material.

The EDS analysis determined the spatial distribution of chemical elements and confirmed the presence of areas of bone regeneration (Figure 6). In the control group, there were no areas of bone regeneration in any of the samples, while in groups $\mathrm{RBC}$ and $\mathrm{RBC}+\mathrm{DPSC}$ calcified nodules were identified sparsely distributed over the defect area.

\section{Discussion}

There is a constant search for replacements of bone tissue to treat bone defects and lesions. In this context, the development of biomaterials and techniques of cell therapy raises promising perspectives in this area. Several studies have been published reporting bone reconstruction using stem cell transplantation [5,7]. The ideal replacement would be a biomaterial with osteoconductive and/ or osteogenic and/or osteoinductive properties [8]. In the present study, we evaluated the biomaterial $\mathrm{RBC}$ in association with DPSC.

The choice for DPSC was mainly due to promising results reported $[4,5,9]$, where the capacity of proliferation, plasticity, and tissue regenerative potential of DPSC was confirmed. However, it should be emphasized that the collection of the tissue from decidual teeth has a limited time due to mixed dentition [10]. A way to bypass this limitation is the cryopreservation of the cells, the pulp tissue, or even the whole tooth [11].

Calcium deposition was observed in all cultures using osteogenic medium and biomaterial extract, confirming the differentiation process. It is a positive result that the biomaterial did not inhibit cell differentiation. This work did not assess the potential of biomaterials for inducing differentiation in undifferentiated stem cells. However, Beloti 
Figure 4. Histological analysis of bone defects by optical microscopy.
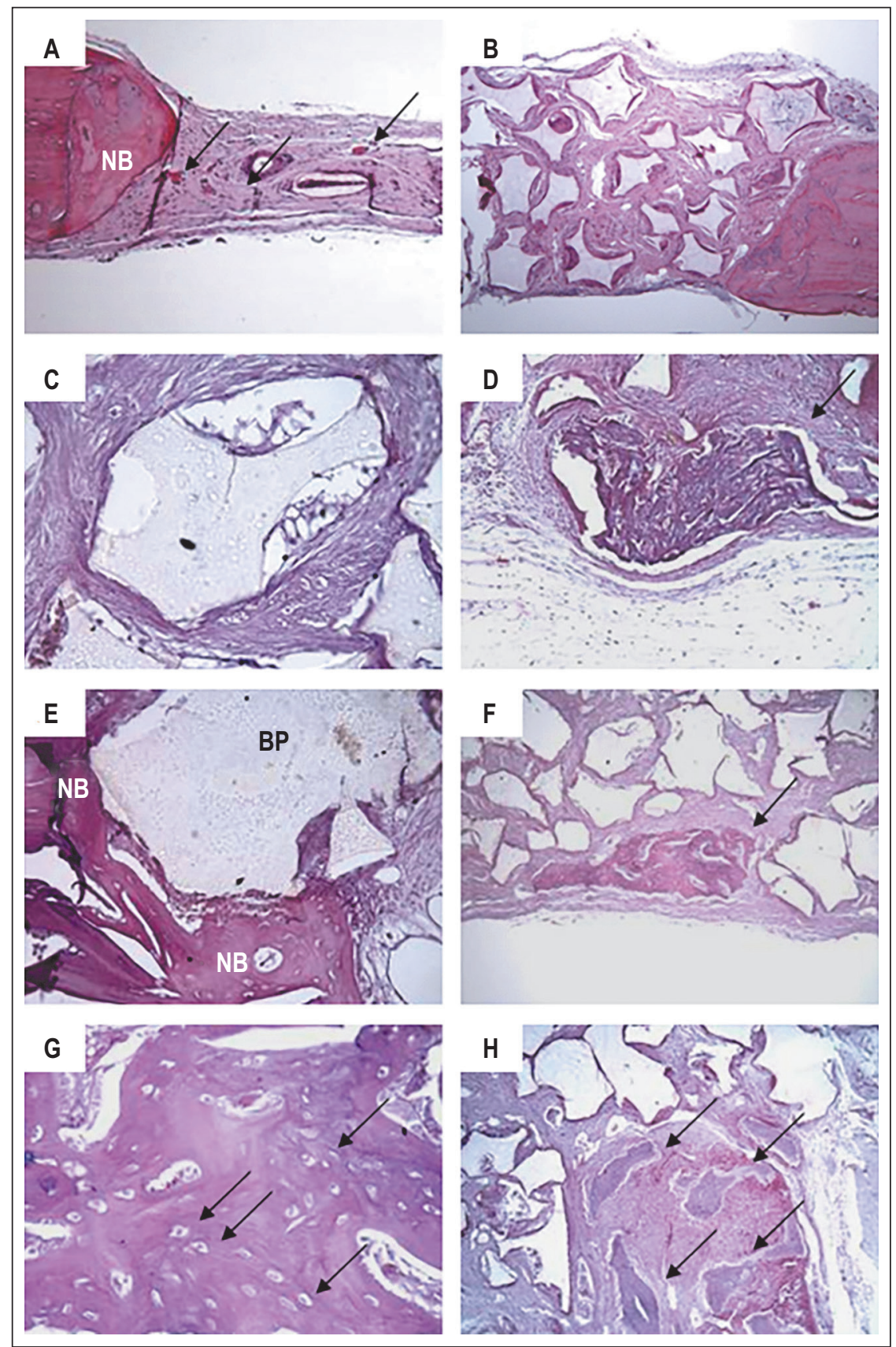

(A) The section made from one animal from the control group euthanized at day 60 after defect induction, showing a discrete bone neoformation restricted to the margins (NB), with a decrease in thickness in the defect region. Blood vessels near the edges (arrows). (B-E) Section obtained from animals of the RBC group. (B) Maintenance of the anatomy before the defect at day 30 after defect induction. (C) Particles of biomaterial with spiked edges blocking contact from the tissue with the surface of the biomaterial at day 60 after defect induction. (D) Presence of calcification points in the center of the lesion (arrow) at day 60 after defect induction. (E) Signs of osseointegration of the biomaterial's particles (BP) with the newly-formed bone (NB) at day 60 after defect induction. (F-H) Sections were obtained from the RBC+DPSC group. (F) Calcification area in the center of the lesion (arrow) at day 15 after defect induction. (G) Details of figure F, showing the maturation aspect of the tissue, with the presence of several osteocytes (arrows). (H) The region with multiple individual calcification areas (arrows) 30 days after defect induction. Hematoxylin and eosin staining. Obj: 10x (A and B), 20x (F and H), 40x (D) and 60x (C, E and G). 
Figure 5. Ultra-structural analysis by scanning electron microscopy at day 60 after bone defect induction.
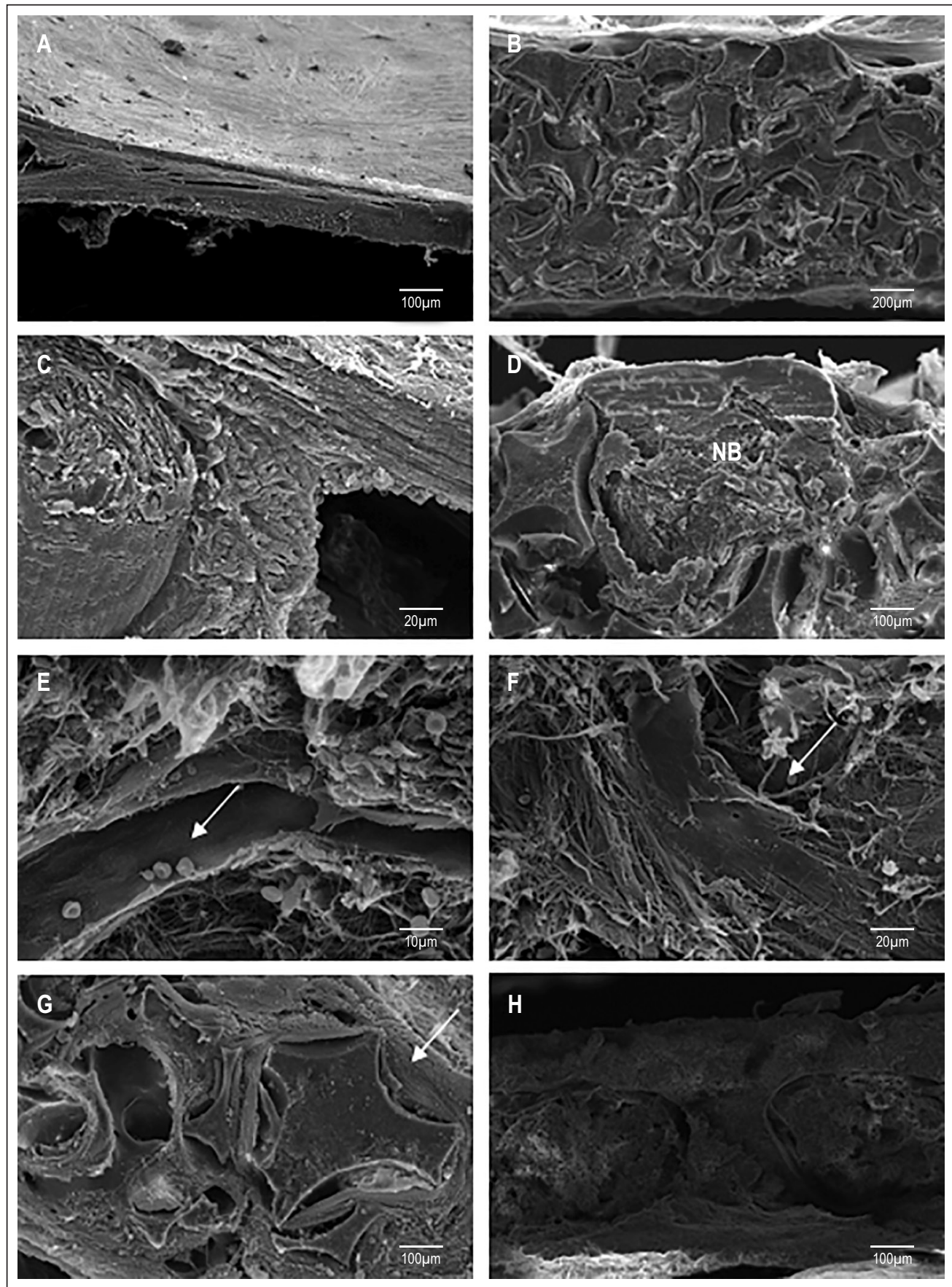

(A) Details of the decreased thickness of the bone defect in the control group. (B) View from the bone defect thickness, showing the mechanical filling role of the biomaterial in section obtained from the RBC group. (C) Lesion margin with rounded aspect with no signs of bone neoformation in section obtained from the control group. (D) Presence of calcified region (NB) in section obtained from the RBC+DPSC group. (E) Longitudinal section of a blood vessel with erythrocytes (arrow), demonstrating vascularization, but with the surrounding tissues not calcified in the sample from the RBC group. (F) Fibrous aspect from the tissue in the center of the bone with bundles of newly formed fibers (arrow) in the sample from the RBC group. (G) The contact surface between newly formed fibers and the edges of the material (arrow). (H) Micrograph of the intact bone fragment at the same studied area. 
Figure 6. Comparison of bone regeneration between two selected areas in one of the RBC+DPSC samples by electron dispersive spectrometry.
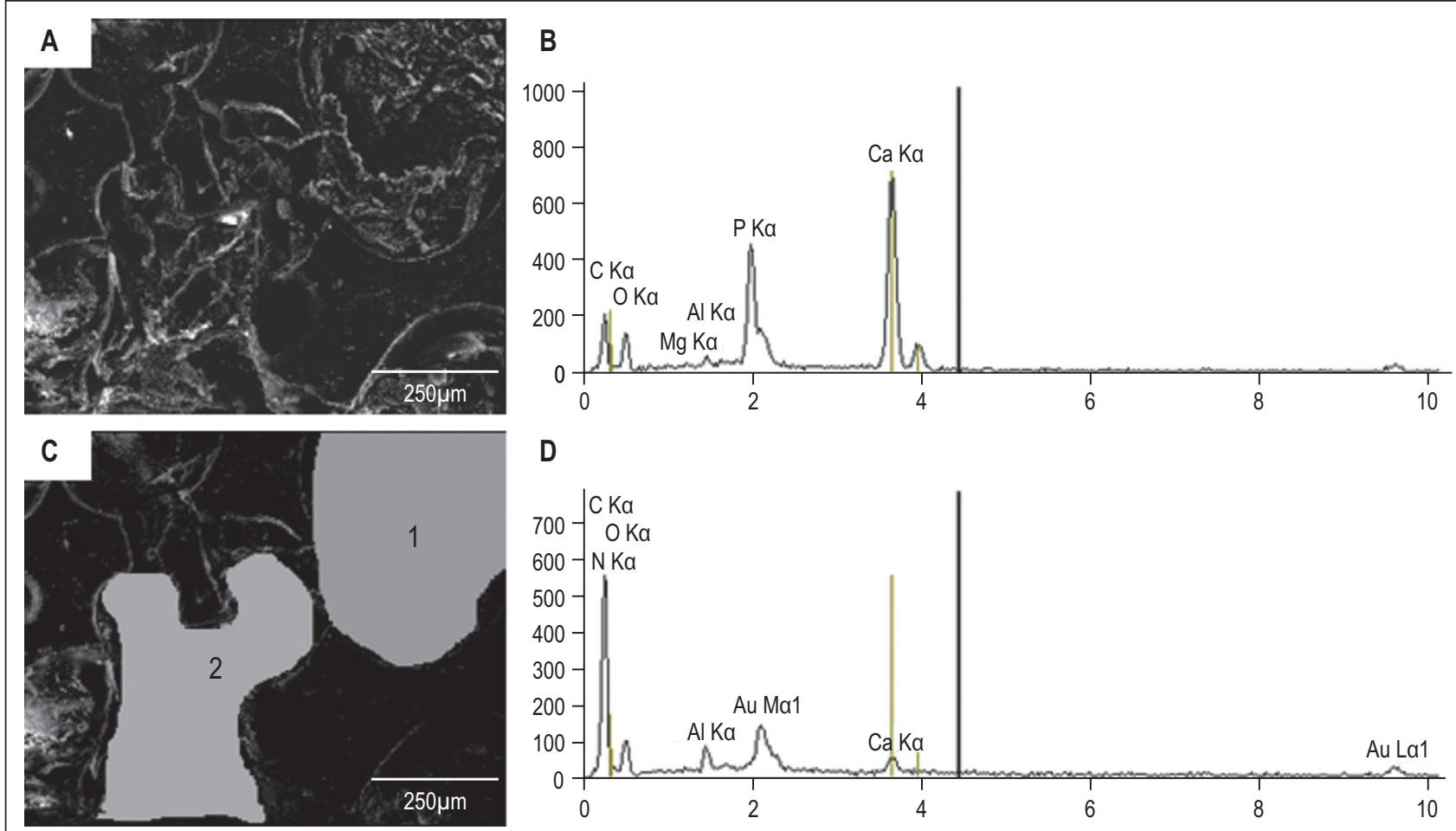

D

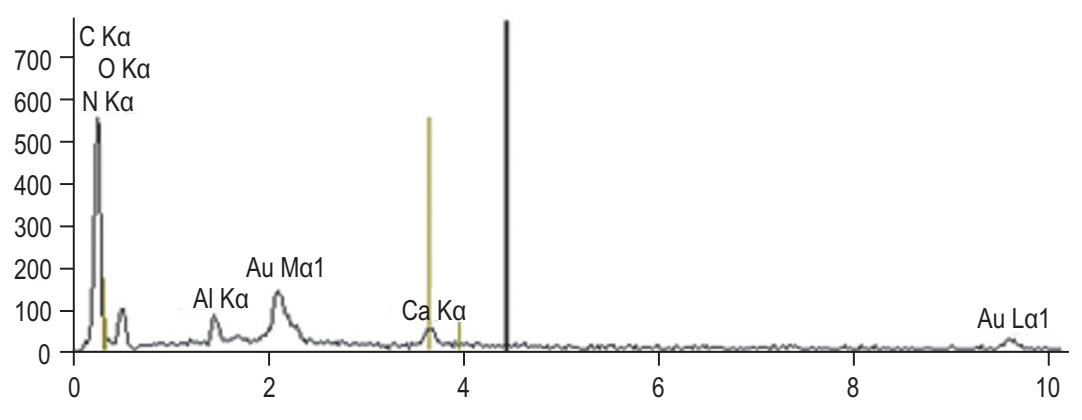

(A) Micrograph of the entire analyzed area by SEM. (B) Quantitative analysis of the chemical elements present in area 1. (C) The two selected areas (in gray) for analysis do not contain biomaterial particles. (D) Quantitative analysis of the chemical elements present in area 2. A bone neoformation in area 1 is confirmed by its chemical composition.

and colleagues [12] found no signs of osteogenic differentiation in cultures associated with polyurethane discs derived from Ricinus communis.

The choice for the RBC biomaterial was due to its vegetal origin, which avoids possible complications related to the use of biomaterials from human and animal sources, such as contamination and resistance to the user due to the patient's principles. Also, RBC is derived from castor oil, a low-cost renewable resource found in abundance in Brazil and other countries [13]. Moreover, there is no in vivo report assessing its association with DPSC in bone regeneration.

The analysis by digital radiographs showed that the control groups presented significantly lower values of gray levels and $\mathrm{CV}$ than the groups with the biomaterial, proving the biomaterial's role as a mechanical support for the regenerating tissues. Results of gray level intensities express an increased general radiopacity of the area, while $\mathrm{CV}$ indicates a greater heterogeneity of the analyzed tissues. The analysis at 15 days demonstrated higher gray levels and $\mathrm{CV}$ values for $\mathrm{RBC}+\mathrm{DPSC}$ groups than for the others $(p<0.05)$. RBC+DPSC groups showed more radiopaque pixels that may suggest early-onset bone regeneration, possibly due to the influence of DPSC on the tissues. This increase in radiopacity was also observed by Del Carlo and colleagues [14], in animals that received polyurethane from castor oil associated with aspirate from autologous bone marrow to regenerate bone defects.

Confirming these results, macro and microscopic assessment of this group at the same time point presented also improved regeneration. However, it is worth mentioning that radiograph analyses have limitations and it is not possible to assert the type of tissue formed in the bone defects, although 
radiographic analysis can be found in several reports on bone regeneration [15]. The samples may become more radiopaque due to an increase in soft tissue instead of bone tissue formation. In any case, a potential tissue formation may represent a regenerative activity, which may be confirmed and better understood when investigated in combination with microscopic analyses, as carried out in this study.

Association of analysis between optical microscopy and SEM allows assessment of the histological characteristic and tissue microstructures, identifying ossified regions and the interaction between the tissue and the biomaterial [16]. Although the assessment by SEM was qualitative, in case of doubt whether a structure was truly the result of bone regeneration, we performed analyses by EDS to identify and quantify the chemical elements present in the structure and whether those elements present in the right proportion corresponded to regenerating a bone tissue.

The biomaterial fulfilled the basic requirements for biocompatibility [16]. No significant inflammatory infiltrate was found in the samples and it served as support for newly-formed tissues, allowing the defect region to keep its architecture. It was evident the difference in tissue volume between the control group and the groups with the biomaterial. Some regeneration areas showed interaction with the particle's surface of the biomaterial, indicating some type of influence of the biomaterial on the process. It was also detected a close contact between them, possibly meaning a potential for osseointegration. The action of the biomaterials' surface over bone regeneration is well described in the work of Olivier and colleagues [17]. The irregular shape of the RBC particles may hamper bone regeneration due to a decrease in the contact surface, where empty spaces without tissue formation would remain, as suggested before [2].

When comparing RBC+DPSC and RBC groups, samples showed very similar characteristics, though qualitative differences were found. In the group $\mathrm{RBC}+\mathrm{DPSC}$, ossified areas happened earlier, from day $15 \mathrm{on}$, and exhibited larger size at certain points, which may indicate a positive effect of the DPSC on the initial tissue regeneration. As previously reported by Giannoni and colleagues [18], cells could have a regenerative activity for a limited time, decrease over time due to the low cellular level of bone tissue and the difficult tissue vascularization.

An increased vascularization over time was observed in all groups; however, the defect that received biomaterial presented blood vessels overall in all-time points, while in the control group vessels were found predominantly near the margin. This is probably a positive factor, though there is no guarantee of bone regeneration, as previously reported by Koob and colleagues [19].

Although there is a lack of consensus about the ideal degradation time of biomaterials, they ideally should be gradually replaced by the newlyformed bone tissue or incorporated into the tissue's receptor [17]. In our study, the RBC particles did not undergo any volume alteration. This finding is in line with most reports about polymeric biomaterials derived from castor oil [14].

Nevertheless, one report showed signs of particle reabsorption and replacement by bone tissue [20]. The time-frame of the present work did not allow to state that the biomaterial has a null degradation, nor it allows to state that it is slower than the ideal since full regeneration of the effect did not take place. Maintenance of the biomaterial at the defect site might be an advantage in situations as craniofacial bone defects since maintenance of the graft volume is important for esthetical purposes.

\section{Conclusion}

Finally, we conclude that the biomaterial tested in this study show biocompatibility in vitro and in vivo and promising use in association with DPSC. Further studies should be carried out to extend the knowledge of RBC applications in regenerative medicine.

\section{Acknowledgments}

The authors wish to thank Claudio Pereira Figueira (FIOCRUZ, Bahia, Brazil) for technical 
assistance with the SEM images and analysis and Marcelo Borges Velame (Salvador, Brazil) and Viviane Almeida Sarmento (UFBA, Salvador, Brazil) for assistance with the X-ray images.

\section{References}

1. Mauffrey C, Barlow BT, Smith W. Management of segmental bone defects. J Am Acad Orthop Surg. 2015; 23(3):143-153.

2. Ghanaati S, Barbeck M, Orth C, Willershausen I, Thimm BW, Hoffmann C, et al. Influence of beta-tricalcium phosphate granule size and morphology on tissue reaction in vivo. Acta Biomater. 2010; 6(12): 4476-4487.

3. Szpalski C, Barbaro M, Sagebin F, Warren SM. Bone tissue engineering: current strategies and techniques-part II: Cell types. Tissue Eng Part B Rev. 2012; 18(4): 258269.

4. Kaneko T, Gu B, Sone PP, Zaw SYM, Murano H, Zaw ZCT, et al. Dental pulp tissue engineering using mesenchymal stem cells: a review with a protocol. Stem Cell Rev Rep. 2018; 14(5): 668-676.

5. Dave JR, Tomar GB. Dental tissue-derived mesenchymal stem cells: applications in tissue engineering. Crit Rev Biomed Eng. 2018; 46(5): 429-468.

6. Jesus AA, Soares MBP, Soares AP; Nogueira RC, Guimaraes ET, Araujo TM, et al. Collection and culture of stem cells derived from dental pulp of deciduous teeth: technique and clinical case report. Dental Press J Orthod. 2011; 16(6): 111-7.

7. Yamada Y, Hara K, Nakamura S, Ueda M, Ito K, Nagasaka T. Minimally invasive approach with tissue engineering for severe alveolar bone atrophy case. Int $\mathrm{J}$ Oral Maxillofac Surg. 2013; 42(2): 260-3.

8. Reichert C, Al-Nawas B, Smeets R, Kasaj A, Götz W, Klein, MO. In vitro proliferation of human osteogenic cells in presence of different commercial bone substitute materials combined with enamel matrix derivatives. Head Face Med. 2009; 5: 23.

9. Lymperi S, Ligoudistianou C, Taraslia V, Kontakiotis E, Anastasiadou E. Dental Stem Cells and their Applications in Dental Tissue Engineering. Open Dent J. 2013; 7: 76-81.

10. Nolla CM. The development of the permanent teeth. J Dent Child. 1960; 27: 254-66.
11. Perry BC, Zhou D, Wu X, Yang FC, Byers MA, Chu TM, et al. Collection, cryopreservation, and characterization of human dental pulp-derived mesenchymal stem cells for banking and clinical use. Tissue Eng Part C Methods. 2008; 14(2): 149-56.

12. Beloti MM, Oliveira PT, Tagliani MM, Rosa AL. Bone cell responses to the composite of Ricinus communis polyurethane and alkaline phosphatase. J Biomed Mater Res A. 2008; 84(2): 435-41.

13. Leite FR, Ramalho LT. Bone regeneration after demineralized bone matrix and castor oil (Ricinus Communis) polyurethane implantation. J Appl Oral Sci. 2008; 16(2): 122-26.

14. Del Carlo RJ, Kawata D, Viloria MIV, Oliveira DR, Silva AS, Marchesi DR, et al. Castor oil plant polymer and calcium associated or not to autogenous bone marrow in bone gaps repair. Ciênc Rural. 2003; 33(6): 1081-88.

15. Martins R, Kinoshita AMO, Carvalho NTA, Guimarães SAC. Comparative study of bone response guided tissue regeneration technique - macroscopic evaluation. Part 1. FULL Dentistry in Science. 2010; 1(3): 224-30.

16. Nóbrega FS, Selim MB, Arana-Chavez VE, Correa L, Ferreira MP, Zoppa ALV. Histologic and immunohistochemical evaluation of biocompatibility of castor oil polyurethane polymer with calcium carbonate in equine bone tissue. Am J Vet Res. 2017; 78(10): 1210-1214.

17. Olivier V, Faucheux N, Hardouin P. Biomaterial challenges and approaches to stem cell use in bone reconstructive surgery. Drug Discov Today. 2004; 9(18): 803-11.

18. Giannoni P, Scaglione S, Daga A, Ilengo C, Cilli M, Quarto R. Short-time survival and engraftment of bone marrow stromal cells in an ectopic model of bone regeneration. Tissue Eng Part A. 2009; 16(2): 489-99.

19. Koob S, Torio-Padron N, Stark B, Hannig C, Stankovic Z, Finkenzeller G. Bone formation and neovascularization mediated by mesenchymal stem cells and endothelial cells in critical-sized calvarial defects. Tissue Eng Part A. 2011; 17(3-4): 311-21.

20. Mendoza-Barrera C, Meléndez-Lira M, Altuzar V, Tomás SA. Ricinus communis-based biopolymer and epidermal growth factor Regulations on bone defect repair: A rat tibia model. Rev Sci Instrum. 2003; 74(1): 390-2. 